\title{
THE ANALYSIS OF IMAGES IN N-POINT GRAVITATIONAL LENS BY METHODS OF ALGEBRAIC GEOMETRY
}

\author{
Albert T. KotvytskiY ${ }^{a, b, *}$, Semen D. BronzA ${ }^{b}$, \\ VOLODYMYr Yu. SHABLENKO ${ }^{a}$ \\ ${ }^{a}$ Karazin Kharkov National University, Svobody Square 4, Kharkiv, 61022, Ukraine \\ ${ }^{b}$ Ukrainian State University of Railway Transport, Feierbakh Square 7, 61050, Kharkiv, Ukraine \\ * corresponding author: kotvytskiy@gmail.com
}

ABstract. This paper is devoted to the study of images in $N$-point gravitational lenses by methods of algebraic geometry. In the beginning, we carefully define images in algebraic terms. Based on the definition, we show that in this model of gravitational lenses (for a point source), the dimensions of the images can be only 0 and 1 . We reduce it to the fundamental problem of classical algebraic geometry - the study of solutions of a polynomial system of equations. Further, we use well-known concepts and theorems. We adapt known or prove new assertions. Sometimes, these statements have a fairly general form and can be applied to other problems of algebraic geometry. In this paper, the criterion for irreducibility of polynomials in several variables over the field of complex numbers is effectively used. In this paper, an algebraic version of the Bezout theorem and some other statements are formulated and proved. We have applied the theorems proved by us to study the imaging of dimensions 1 and 0 .

KEYWORDS: gravitational lense, images, algebaric geometry, resultant.

\section{INTRODUCTION}

In modern astrophysics, gravitational lensing has been transformed from an effect that confirms the general theory of relativity to the research tool. Gravitational lensing is used to study both stellar systems and planets in them, and galaxies and systems of galaxies. Even the cosmological parameters of the entire metagalaxy are investigated.

From this point of view, it seems rather strange that until now a complete analytical description has been performed only for the simplest lenses - axially symmetric lenses (see for example [1] or straight infinite cosmic strings [2].

To analyze fairly simple 2-point gravitational lenses, only approximate or numerical methods are used [3, 4,

In this paper, the authors continue the analytic study of N-point gravitational lenses by methods of algebraic geometry [5]

In physics, the concept of "image in a gravitational lens" is understood intuitively and is usually not determined. However, the absence of a definition can lead to ambiguous understanding of the concept and a different interpretation of some results, for example, the theorem on the odd number of images [9, 10]. On the other hand, the terminology developed in algebraic geometry makes it possible to pinpoint the concept of an image in a gravitational lens. On this basis it is possible to formulate a number of statements.

\section{THE PHYSICAL FORMULATION OF THE PROBLEM FROM \\ AN ALGEBRAIC POINT OF VIEW}

For the model of a plane gravitational lens, we can write the equation that connects the coordinates of the source (the radius vector $\vec{y}$ ) and the image coordinates (radius vector $\vec{x}$ ), see [9, 10]

$$
\vec{y}=\vec{x}-\vec{\alpha},
$$

where $\vec{\alpha}$ is the total angle of deflection of the light beam in the plane of the lens. In the case of an N-point gravitational lens, the deflection angle is determined by the following expression:

$$
\vec{\alpha}=\sum_{i=1}^{N} m_{i} \frac{\vec{x}-\overrightarrow{l_{i}}}{\left|\vec{x}-\vec{l}_{i}\right|^{2}},
$$

where $m_{i}$ are dimensionless masses whose position in the plane of the lens is determined by the radius vectors $\vec{l}_{i}$. We have that holds $\sum_{i=1}^{N} m_{i}=1$.

Equation (1) with allowance for (2) in coordinate form has the form

$$
\left\{\begin{array}{l}
\left(x_{1}-\sum_{i=1}^{N} m_{i} \frac{x_{1}-a_{i}}{\left(x_{1}-a_{i}\right)^{2}+\left(x_{2}-b_{i}\right)^{2}}\right)-y_{1}=0 \\
\left(x_{2}-\sum_{i=1}^{N} m_{i} \frac{x_{2}-b_{i}}{\left(x_{1}-a_{i}\right)^{2}+\left(x_{2}-b_{i}\right)^{2}}\right)-y_{2}=0
\end{array}\right.
$$

where $a_{i}$ and $b_{i}$ are the coordinates of the radius-vector $\vec{l}_{i}$ i.e. $\vec{l}_{i}=\left(a_{i}, b_{i}\right)$.

From an algebraic point of view, system (3) is a system of two rational equations (over a field of real numbers) from two unknowns, which are given in Cartesian coordinates on the $R^{2}$ plane. The system (3) will be considered, just above the field of complex numbers $C$, while we denote it by $(3$ a). The set of solutions of system (3) is obviously the set of real solutions of system $(3 a)$. We note that all the coefficients of the equations of system $(3 \mathrm{a})$ are real. 
In terms of algebraic geometry, the image of a source in an $\mathrm{N}$-point gravitational lens can be defined as follows:

Definition. An image of a point source in an N-point gravitational lens will be called the real solution of system (3a) without regard to multiplicity. The set of images is the set of different real solutions of this system.

\section{REDUCTION OF THE PROBLEM TO THE FUNDAMENTAL PROBLEM OF CLASSICAL ALGEBRAIC GEOMETRY}

The main problem of classical algebraic geometry is the problem of studying systems of polynomial equations.

Let us investigate the set of solutions of system (3a). To do this, we transform the equations of the system to a polynomial form

$$
\left\{\begin{array}{l}
F_{1}=\left(x_{1}-y_{1}\right) \prod_{i=1}^{N} h_{i}-\sum_{j=1}^{N} m_{j}\left(x_{1}-a_{j}\right) \prod_{i=1, i \neq j}^{N} h_{i}=0 \\
F_{2}=\left(x_{2}-y_{2}\right) \prod_{i=1}^{N} h_{i}-\sum_{i=1}^{N} m_{i}\left(x_{2}-b_{i}\right) \prod_{i=1, i \neq j}^{N} h_{i}=0
\end{array}\right.
$$

where $h_{i}=\left(x_{1}-a_{i}\right)^{2}+\left(x_{2}-b_{i}\right)^{2}, i=1,2, \ldots, N$.

The polynomial form of the equations in system (4) is necessary for its investigation by methods of algebraic geometry.

We shall consider the equations of the system (4) over the field $C$ of complex numbers in the affine coordinate system $C^{2}$. The system $(4)$ is not equivalent to the system (3a), but it follows from it. The set of solutions of the system (3a) can be obtained from the set of solutions of the system (4). For this, by removing solutions from it in which the system $(3 a)$ is not defined. These solutions are pairs of numbers that are the coordinates of the point masses. Indeed, we directly verify that the points with coordinates $\left(a_{i}, b_{j}\right), i=1, \ldots, N$, is a solution of the system (4), but the system (3a), in these points is not defined.

Let $f_{1}$ and $f_{2}$ be the left-hand sides of the first and second equations of system (3a), $M\left(f_{1}, f_{2}\right)$ be the solution set of system $(3 \mathrm{a}), V\left(F_{1}, F_{2}\right)$ be the solution set of system (4), and $\operatorname{Re} V\left(F_{1}, F_{2}\right) \subset V\left(F_{1}, F_{2}\right)$ the subset of its real solutions, then we have

$$
M\left(f_{1}, f_{2}\right)=\operatorname{Re} V\left(F_{1}, F_{2}\right) /\left\{\cup\left(a_{i}, b_{i}\right)\right\} .
$$

From the theorem on the structure of the set of solutions of a system of polynomial equations, see [11] it follows that the set $V\left(F_{1}, F_{2}\right)$ can be represented in the form

$$
V\left(F_{1}, F_{2}\right)=\left(V^{0}\left(F_{1}, F_{2}\right)\right) \cup\left(V^{1}\left(F_{1}, F_{2}\right)\right),
$$

where $V^{1}\left(F_{1}, F_{2}\right)$ is the set of solutions depending on a single parameter, and $V^{0}\left(F_{1}, F_{2}\right)$ is the discrete set of solutions of system (3a).
The set $V^{0}\left(F_{1}, F_{2}\right)$ is obviously discrete and, moreover, finite. The sets have dimension dim $V^{0}\left(F_{1}, F_{2}\right)=0$ and $\operatorname{dim} V^{1}\left(F_{1}, F_{2}\right)=1$.

\section{Study of The Set $V^{1}\left(F_{1}, F_{2}\right)$ (EXTENDED SOLUTIONS)}

A number of theorems, which allow us to determine if the set $V^{1}\left(F_{1}, F_{2}\right)$ is empty, see, for example, [12. 13. In [5], we give an algorithm that allows us to describe this set analytically, if it is not empty. If the set $V^{1}\left(F_{1}, F_{2}\right)$ is not empty, then the equations of system (3) are said to have a common component. The equation of the common component can be obtained from the analytical description of the set $V^{1}\left(F_{1}, F_{2}\right)$.

\subsection{1-POINT LENS (SCHWARZSCHILD LENS)}

We apply the theorems presented in the Appendix for constructing the set $V^{1}\left(F_{1}, F_{2}\right)$, in the case of a single-point gravitational lens.

Let the 1 -point lens have coordinates $a_{1}=0, b_{1}=0$. Let $L: R_{Y}^{2} \rightarrow R_{X}^{2}$ be the transformation from the plane of the source to the plane of the lens, determined by the system of equations

$$
\left\{\begin{array}{l}
y_{1}=x_{1}-\frac{x_{1}}{x_{1}^{2}+x_{2}^{2}} \\
y_{2}=x_{2}-\frac{x_{2}}{x_{1}^{2}+x_{2}^{2}} .
\end{array}\right.
$$

Equations of the system are defined for all points such that $x_{1}^{2}+x_{2}^{2} \neq 0$, that is, except for the origin of the point $O(0,0)$. At the origin, the inverse mapping is not defined. But if we transform system (7) to polynomial form

$$
\left\{\begin{array}{l}
\left(x_{1}^{2}+x_{2}^{2}\right)\left(x_{1}-y_{1}\right)-x_{1}=0 \\
\left(x_{1}^{2}+x_{2}^{2}\right)\left(x_{2}-y_{1}\right)-x_{2}=0
\end{array}\right.
$$

then the inverse transformation of $L^{-1}: R_{X}^{2} \rightarrow R_{Y}^{2}$ is completely determined by the system of equations

$$
\left\{\begin{array}{l}
x_{1}^{3}+x_{1} x_{2}^{2}-x_{1}^{2} y_{1}-x_{2}^{2} y_{1}-x_{1}=0 \\
x_{1}^{2} x_{2}+x_{2}^{3}-x_{1}^{2} y_{2}-x_{2}^{2} y_{2}-x_{2}=0
\end{array}\right.
$$

We calculate the result $R_{1}$ by the variable $x_{1}$ for which we represent the equation in lexicographic form

$$
\left\{\begin{array}{l}
x_{1}^{3}-y_{1} x_{1}^{2}+\left(x_{2}^{2}-1\right) x_{1}-x_{2}^{2} y_{1}=0, \\
\left(x_{2}-y_{2}\right) x_{1}^{2}+x_{2}^{3}-x_{2}^{2} y_{2}-x_{2}=0 .
\end{array}\right.
$$

Result by degree $x_{1}$ has the form

$$
R_{1}=\left|\begin{array}{ccccc}
1 & r_{12} & r_{13} & r_{14} & 0 \\
0 & 1 & r_{12} & r_{13} & r_{14} \\
r_{21} & 0 & r_{23} & 0 & 0 \\
0 & r_{21} & 0 & r_{23} & 0 \\
0 & 0 & r_{21} & 0 & r_{23}
\end{array}\right|
$$

where $r_{12}=-y_{1}, r_{13}=x_{2}^{2}-1, r_{14}=-x_{2}^{2} y_{1}, r_{21}=$ $x_{2}-y_{2}, r_{23}=x_{2}^{3}-x_{2}^{2} y_{2}-x_{2}$. 
We have

$$
R_{1}=-x_{1}^{3} y_{1}^{2}+x_{2}^{2} y_{1}^{2} y_{2}+x_{2} y_{1}^{2}-x_{2}^{3} y_{2}^{2}+x_{2}^{2} y_{2}^{3} \text {. }
$$

In order for the system equations (7) to have a common component, we need $R_{1} \equiv 0$.

Applying Theorem 5 a for the decomposition of $R_{1}$ on indecomposable components, we have

$$
x_{2}\left(-\left(y_{1}^{2}+y_{2}^{2}\right) x_{2}^{2}+y_{2}\left(y_{1}^{2}+y_{2}^{2}\right) x_{2}+y_{1}^{2}\right) \equiv 0 .
$$

The equation is divided into two equations

$$
\begin{gathered}
x_{2} \equiv 0 \\
-\left(y_{1}^{2}+y_{2}^{2}\right) x_{2}^{2}+y_{2}\left(y_{1}^{2}+y_{2}^{2}\right) x_{2}+y_{1}^{2} \equiv 0 .
\end{gathered}
$$

Each of the equations is considered to be a polynomial of the variable $x_{2}$.

A polynomial is identically equal to zero if and only if all its coefficients are equal to zero.

From here there is a system of equations

$$
\left\{\begin{array}{l}
y_{1}^{2}+y_{2}^{2}=0 \\
y_{1}^{2}=0 .
\end{array} .\right.
$$

Next we have that $y_{1}=0, y_{2}=0$. Substituting in (7) we have

$$
\left\{\begin{array} { l } 
{ x _ { 1 } - \frac { x _ { 1 } } { x _ { 1 } ^ { 2 } + x _ { 2 } ^ { 2 } } = 0 , } \\
{ x _ { 2 } - \frac { x _ { 2 } } { x _ { 1 } ^ { 2 } + x _ { 2 } ^ { 2 } } = 0 }
\end{array} \Rightarrow \left\{\begin{array}{l}
x_{1}\left(1-\frac{1}{x_{1}^{2}+x_{2}^{2}}\right)=0 \\
x_{2}\left(1-\frac{1}{x_{1}^{2}+x_{2}^{2}}\right)=0
\end{array}\right.\right.
$$

The system 16 decomposes into three systems and one equation:

$$
\begin{aligned}
& \left\{\begin{array}{l}
x_{1}=0, \\
x_{2}=0,
\end{array}\right. \\
& \left\{\begin{array}{l}
x_{1}=0, \\
1-\frac{1}{x_{1}^{2}+x_{2}^{2}}=0,
\end{array}\right. \\
& \left\{\begin{array}{l}
1-\frac{1}{x_{1}^{2}+x_{2}^{2}}=0, \\
x_{2}=0,
\end{array}\right. \\
& 1-\frac{1}{x_{1}^{2}+x_{2}^{2}}=0 .
\end{aligned}
$$

System 17a has a solution $x_{1}=0, x_{2}=0$ but this solution is not a solution of system (7), since the system equations at the point $O(0,0)$ are not defined.

The system $17 \mathrm{~b}$ has two solutions $x_{1}=0, x_{2}=$ \pm 1 . 0 .

The system $17 \mathrm{c}$ has two solutions $x_{1}= \pm 1, x_{2}=$

We have the transformation of equation 18 into

$$
x_{1}^{2}+x_{2}^{2}-1=0
$$

Equation 19 is the equation of an individual circle in the plane $X$ with a center at the point $O(0,0)$.
The solution of systems $(17 \mathrm{~b})$ and $(17 \mathrm{c})$ satisfies equation (19).

The solution of system (8) is the coordinates of the points of single circle with center at the point $O(0,0)$.

Equation 19 is the equation of the general component, hence the set

$$
V^{1}\left(F_{1}, F_{2}\right)=\left\{x_{1}, x_{2} \mid x_{1}^{2}+x_{2}^{2}-1=0\right\} .
$$

In the same way, we compute the resultant $R_{2}$. By virtue of the symmetry of variables, we have the same solution.

\subsection{2-POINT LENS}

We research a two-point gravitational lens with equal masses $m_{1}=m_{2}=\frac{1}{2}$.

The masses are on the abscissa at a distance $a$ from the origin of coordinates.

In this case, system looks like this:

$$
\left\{\begin{array}{l}
y_{1}=x_{1}-\frac{1}{2} \frac{x_{1}-a}{\left(x_{1}-a\right)^{2}+x_{2}^{2}}-\frac{1}{2} \frac{x_{1}+a}{\left(x_{1}+a\right)^{2}+x_{2}^{2}}, \\
y_{2}=x_{2}-\frac{1}{2} \frac{x_{2}}{\left(x_{1}-a\right)^{2}+x_{2}^{2}}-\frac{1}{2} \frac{x_{2}}{\left(x_{1}+a\right)^{2}+x_{2}^{2}} .
\end{array}\right.
$$

We transform the equation of system (21) into a polynomial form, and represent the obtained polynomials $F_{1}$ and $F_{2}$ in lexicographic form with increasing degrees of variable $x_{1}$ :

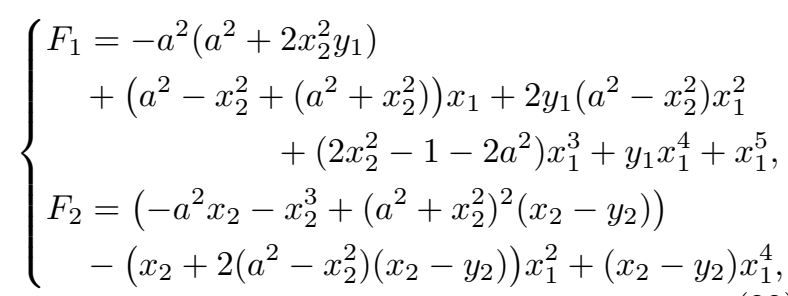

We will remove from the system the variable $x_{1}$, using the resultant $R_{1}=R\left(F_{1}, F_{2}\right)$.

Sylvester matrix $S_{1}=S\left(F_{1}, F_{2}\right)$ has order (for $x_{1}$ ) $\operatorname{deg} F_{1}+\operatorname{deg} F_{2}=9$.

Because $R_{1}=\operatorname{det} S_{1}$, we have

$$
\begin{gathered}
R_{1}=4 a^{4} x_{2}^{2}\left(a^{2}+x_{2}^{2}\right)\left(-a^{2} y_{2}^{3}\right. \\
+\left(a^{2} y_{2}^{2}-y_{2}^{2}-4 a^{4} y_{2}^{2}-4 a^{2} y_{2}^{4}\right) x_{2} \\
+\left(-4 a^{2} y_{2}+4 a^{4} y_{2}-4 a^{6} y_{2}-y_{1}^{2} y^{2}\right. \\
-4 a^{2} y_{1}^{2} y_{2}+8 a^{4} y_{1}^{2} y_{2}-4 a^{2} y_{1}^{2} y_{2} \\
\left.\quad-5 y_{2}^{3}+4 a^{2} y_{2}^{3}-8 a^{4} y_{2}^{3}-4 a^{2} y_{2}^{5}\right) x_{2}^{2} \\
+\left(-4 a^{4}+4 a^{6}+y_{1}^{2}+4 a^{2} y_{1}^{2}\right. \\
-8 a^{4} y_{1}^{2}+4 a^{2} y_{1}^{4}+y_{2}^{2}-12 a^{2} y_{2}^{2} \\
\left.\quad+8 a^{4} y_{2}^{2}-8 y_{1}^{2} y_{2}^{2}-8 y_{2}^{4}+4 a^{2} y_{2}^{4}\right) x_{2}^{3} \\
-4\left(a^{4} y_{2}-a^{2} y_{2}-y_{1}^{2} y_{2}-2 a^{2} y_{1}^{2} y_{2}\right. \\
\left.\quad+y_{1}^{4} y_{2}-y_{2}^{3}+2 a^{2} y_{2}^{3}+2 y_{1}^{2} y_{2}^{3}+y_{2}^{5}\right) x_{2}^{4} \\
\left.+4\left(a^{4}-2 a^{2} y_{1}^{2}+y_{1}^{4}+2 a^{2} y_{2}^{2}+2 y_{1}^{2} y_{2}^{2}+y_{2}^{4}\right) x_{2}^{5}\right) .
\end{gathered}
$$


In order for the system equation to have a common component, it is sufficient that the objects $R_{1} \equiv 0$.

We have that the equation decomposes into three simple equations and one non-trivial equation.

From the trivial equation $a^{4} \equiv 0, x_{2}^{2} \equiv 0,\left(a^{2}+x_{2}^{2}\right) \equiv$ 0 it follows that their solutions are reduced to 1-lens, or incommensurate.

We have a nontrivial equation

$$
\begin{gathered}
-a^{2} y_{2}^{3}+\left(a^{2} y_{2}^{2}-y_{2}^{2}-4 a^{4} y_{2}^{2}-4 a^{2} y_{2}^{4}\right) x_{2} \\
+\left(-4 a^{2} y_{2}+4 a^{4} y_{2}-4 a^{6} y_{2}-y_{1}^{2} y_{2}^{2}-4 a^{2} y_{1}^{2} y_{2}+8 a^{4} y_{1}^{2} y_{2}\right. \\
\left.-4 a^{2} y_{1}^{2} y_{2}-5 y_{2}^{3}+4 a^{2} y_{2}^{3}-8 a^{4} y_{2}^{3}-4 a^{2} y_{2}^{5}\right) x_{2}^{2} \\
+\left(-4 a^{4}+4 a^{6}+y_{1}^{2}+4 a^{2} y_{1}^{2}-8 a^{4} y_{1}^{2}+4 a^{2} y_{1}^{4}+y_{2}^{2}\right. \\
\left.-12 a^{2} y_{2}^{2}+8 a^{4} y_{2}^{2}-8 y_{1}^{2} y_{2}^{2}-8 y_{2}^{4}+4 a^{2} y_{2}^{4}\right) x_{2}^{3} \\
-4\left(a^{4} y_{2}-a^{2} y_{2}-y_{1}^{2} y_{2}-2 a^{2} y_{1}^{2} y_{2}+y_{1}^{4} y_{2}\right. \\
\left.-y_{2}^{3}+2 a^{2} y_{2}^{3}+2 y_{1}^{2} y_{2}^{3}+y_{2}^{5}\right) x_{2}^{4} \\
+4\left(a^{4}-2 a^{2} y_{1}^{2}+y_{1}^{4}+2 a^{2} y_{2}^{2}+2 y_{1}^{2} y_{2}^{2}+y_{2}^{4}\right) x_{2}^{5}=0 .
\end{gathered}
$$

We equate all coefficients to zero, and have a system of equations

$$
\left\{\begin{array}{l}
-a^{2} y_{2}^{3}=0 \\
a^{2} y_{2}^{2}-y_{2}^{2}-4 a^{4} y_{2}^{2}-4 a^{2} y_{2}^{4}=0 \\
-4 a^{2} y_{2}+4 a^{4} y_{2}-4 a^{6} y_{2}-y_{1}^{2} y_{2}^{2} \\
\quad-4 a^{2} y_{1}^{2} y_{2}-4 a^{2} y_{2}^{5}-4 a^{2} y_{1}^{2} y_{2} \\
\quad-5 y_{2}^{3}+4 a^{2} y_{2}^{3}-8 a^{4} y_{2}^{3}+8 a^{4} y_{1}^{2} y_{2}=0 \\
-4 a^{4}+4 a^{6}+y_{1}^{2}+4 a^{2} y_{1}^{2}-8 a^{4} y_{1}^{2}+4 a^{2} y_{1}^{4}+y_{2}^{2} \\
\quad-12 a^{2} y_{2}^{2}+8 a^{4} y_{2}^{2}-8 y_{1}^{2} y_{2}^{2}-8 y_{2}^{4}+4 a^{2} y_{2}^{4}=0 \\
a^{4} y_{2}-a^{2} y_{2}-y_{1}^{2} y_{2}-2 a^{2} y_{1}^{2} y_{2}+y_{1}^{4} y_{2} \\
\quad-y_{2}^{3}+2 a^{2} y_{2}^{3}+2 y_{1}^{2} y_{2}^{3}+y_{2}^{5}=0 \\
a^{4}-2 a^{2} y_{1}^{2}+y_{1}^{4}+2 a^{2} y_{2}^{2}+2 y_{1}^{2} y_{2}^{2}+y_{2}^{4}=0 .
\end{array}\right.
$$

We have

$$
\left\{\begin{array}{l}
a=0 \\
y_{2}=0 \\
-y_{1}^{2} y_{2}^{2}-5 y_{2}^{3}=0 \\
-y_{1}^{2} y_{2}+y_{1}^{4} y_{2}-y_{2}^{3}+2 y_{1}^{2} y_{2}^{3}+y_{2}^{5}=0 \\
y_{1}^{4}+2 y_{1}^{2} y_{2}^{2}+y_{2}^{4}=0
\end{array}\right.
$$

The system has one solution $a=0, y_{1}=0, y_{2}=0$. Hence this solution reduces the 2-point gravity lens to 1-point.

Similarly we calculate the resultant $R_{2}$ as

$$
\begin{aligned}
& R_{2}=4 a^{4}\left(a-x_{1}\right) x_{1}^{2}\left(a+x_{1}\right)\left(-a^{2} y_{1}^{3}+\right. \\
& \quad+\left(1+a^{2}+4 a^{4}-4 a^{2} y_{1}^{2}-4 a^{2} y_{2}^{2}\right) y_{1}^{2} x_{1} \\
& +\left(-4 a^{2}+4 a^{4}-4 a^{6}+5 y_{1}^{2}+4 a^{2} y_{1}^{2}+8 a^{4} y_{1}^{2}-4 a^{2} y_{1}^{4}\right. \\
& \left.\quad+y_{2}^{2}-4 a^{2} y_{2}^{2}-8 a^{4} y_{2}^{2}-8 a^{2} y_{1}^{2} y_{2}^{2}-4 a^{2} y_{2}^{4}\right) y_{1} x_{1}^{2} \\
& +\left(4 a^{4}+4 a^{6}+\left(-1-12 a^{2}-8 a^{4}+8 y_{1}^{2}+4 a^{2} y_{1}^{2}\right) y_{1}^{2}\right.
\end{aligned}
$$

$$
\begin{gathered}
\left.+\left(-1+4 a^{2}+8 a^{4}+8 y_{1}^{2}+8 a^{2} y_{1}^{2}+4 a^{2} y_{2}^{2}\right) y_{2}^{2}\right) x_{1}^{3} \\
+\left(4 a^{2} y_{1}^{2}+a^{4} y_{1}-y_{1}^{3}-2 a^{2} y_{1}^{3}+y_{1}^{5}-y_{1} y_{2}^{2}\right. \\
\left.+2 a^{2} y_{1} y_{2}^{2}+2 y_{1}^{3} y_{2}^{2}+y_{1} y_{2}^{4}\right) x_{1}^{4} \\
\left.-4\left(a^{4}-2 a^{2} y_{1}^{2}+y_{1}^{4}+2 a^{2} y_{2}^{2}+2 y_{1}^{2} y_{2}^{2}+y_{2}^{4}\right) x_{1}^{5}\right) .
\end{gathered}
$$

We have that the solution of system (21) reduces the 2-point gravity lens to 1-point. Whence

- for the 1-point gravitational lens set we have

$$
V^{1}\left(F_{1}, F_{2}\right)=\left\{x_{1}, x_{2} \mid x_{1}^{2}+x_{2}^{2}-1=0\right\} ;
$$

- for the 2-point gravitational lens set we have

$$
V^{1}\left(F_{1}, F_{2}\right)=\varnothing .
$$

Based on the studies we have carried out above, one can prove that there are no extended objects for $N$-point gravitational lenses, i.e. $V^{1}\left(F_{1}, F_{2}\right)=\varnothing$.

The set $M\left(f_{1}, f_{2}\right)$ can be represented in the form

$$
M\left(f_{1}, f_{2}\right)=M^{0}\left(f_{1}, f_{2}\right) \cup M^{1}\left(f_{1}, f_{2}\right),
$$

where $M^{0}\left(f_{1}, f_{2}\right)=\operatorname{Re} V^{0}\left(F_{1}, F_{2}\right) /\left\{\cup\left(a_{i}, b_{i}\right)\right\}$ and $M^{1}\left(f_{1}, f_{2}\right)=\operatorname{Re} V^{1}\left(F_{1}, F_{2}\right) /\left\{\cup\left(a_{i}, b_{i}\right)\right\}$.

It is known that the set $M^{1}\left(f_{1}, f_{2}\right)$, for a point source in 1-point lens is not empty, see for example [9, 10, 14, coincides with $V^{1}\left(F_{1}, F_{2}\right)$, see [5] and is Einstein ring. But for a point source in symmetric 2-point lens, we proved [5] that the set $M^{1}\left(f_{1}, f_{2}\right)$ is empty and put forward hypothesis: for N-point lens this set is empty for $N>1$.

\section{The Study of the Set $V^{0}\left(F_{1}, F_{2}\right)$ (Point SOLUTIONS)}

To research the set of solutions $V^{0}\left(F_{1}, F_{2}\right)$ of system (3) we use the Bezout theorem, see for example [11. 13, 15.

In most monographs, the authors formulate the Bezout theorem in geometric terms; see for example [11, 12, 15]. One of these theorems is quoted in Appendix.

In 13 Bezout's theorem is formulated in algebraic terms, but for equations given in affine coordinates. This theorem is also quoted in Appendix.

For our purposes, we formulate this theorem in algebraic terms, but for functions given in homogeneous coordinates.

Theorem 1 (Bezout). Let $G_{1}\left(X_{0}: X_{1}: X_{2}\right)$ and $G_{2}\left(X_{0}: X_{1}: X_{2}\right)$ be homogeneous polynomials, $\operatorname{deg} G_{1}\left(X_{0}: X_{1}: X_{2}\right)=n, \operatorname{deg} G_{2}\left(X_{0}: X_{1}: X_{2}\right)=m$ and the resultant $R_{1}\left(G_{1}, G_{2}\right)$, with respect to variable $X_{1}$ not identically equal to zero. Then the resultant $R_{1}\left(G_{1}, G_{2}\right)$ is a homogeneous polynomial with respect to variables $X_{0}$ and $X_{2}$, and $\operatorname{deg} R_{1}\left(G_{1}, G_{2}\right)=n \cdot m$.

Proof. The resultant $R_{1}\left(G_{1}, G_{2}\right)$ is a polynomial in the variables $X_{0}$ and $X_{2}$. We denote it by $F$, and write 
$F=R_{1}\left(G_{1}, G_{2}\right)$. Let us prove that the polynomial $F=F\left(X_{0}, X_{2}\right)$ is homogeneous and of degree $\operatorname{deg} F=$ $n \cdot m$. Really, we have

$$
\begin{array}{r}
F\left(t X_{0}, t X_{2}\right)=R_{1}\left(G_{1}\left(t X_{0}: X_{1}: t X_{2}\right),\right. \\
\left.G_{2}\left(t X_{0}: X_{1}: t X_{2}\right)\right) .
\end{array}
$$

According to Theorem 1a, see Appendix, we have

$$
\begin{aligned}
& R_{1}\left(G_{1}\left(t X_{0}: X_{1}: t X_{2}\right), G_{2}\left(t X_{0}: X_{1}: t X_{2}\right)\right) \\
= & \operatorname{det} \operatorname{Sul}\left(G_{1}\left(t X_{0}: X_{1}: t X_{2}\right), G_{2}\left(t X_{0}: X_{1}: t X_{2}\right)\right) .
\end{aligned}
$$

where the right-hand side of expression 30 is the determinant of the Sylvester matrix. The order of this determinant is $n+m$.

Elements of the Sylvester matrix are coeficies from the lexicographic representation of homogeneous polynomials $G_{1}$ and $G_{2}$. We have that

$$
G_{1}=\sum_{i=0}^{n} a_{i} X_{1}^{n-i} \quad \text { and } \quad G_{2}=\sum_{j=0}^{m} b_{j} X_{1}^{m-j}
$$

The coefficients $a_{i}=a_{i}\left(X_{0}: X_{2}\right)$ and $b_{j}=b_{j}\left(X_{0}\right.$ : $\left.X_{2}\right)$ are homogeneous polynomials.

The degree is $\operatorname{deg} a_{i}\left(X_{0}: X_{2}\right)=i$ and $\operatorname{deg} b_{j}\left(X_{0}\right.$ : $\left.X_{2}\right)=j$, that is, $a_{i}\left(t X_{0}: t X_{2}\right)=t^{i} a_{i}\left(X_{0}: X_{2}\right)$ and $b_{j}\left(t X_{0}: t X_{2}\right)=t^{j} b_{j}\left(X_{0}: X_{2}\right)$.

We multiply every row of the determinant of the Sylvester matrix by the parameter $t$ in some degree. We choose a degree so that all elements of the column are of the same degree with respect to $t$.

We multiply the $i$-th row of the determinant of the Sylvester matrix by $t^{i}$ where $i=1,2, \ldots, m$, and $j$-th row by $t^{j}$ where $j=m+1, m+2, \ldots, m+n$. We take the factor $t^{s}$ from the $s$-th column where $s=1,2, \ldots, m+n$. We denote the total power of $t$ by $S$. We have

$$
\begin{array}{r}
S=\sum_{i=1}^{m+n} i-\sum_{i=1}^{n} i-\sum_{i=1}^{m} i=\frac{(n+m)(n+m+1)}{2} \\
-\frac{n(n+1)}{2}-\frac{m(m+1)}{2}=n m .
\end{array}
$$

In this way,

$$
\begin{gathered}
\operatorname{det} \operatorname{Sul}\left(G_{1}\left(t X_{0}: X_{1}: t X_{2}\right), G_{2}\left(t X_{0}: X_{1}: t X_{2}\right)\right) \\
=t^{n m} \operatorname{det} \operatorname{Sul}\left(G_{1}\left(X_{0}: X_{1}: X_{2}\right)\right. \\
\left.G_{2}\left(X_{0}: X_{1}: X_{2}\right)\right) .
\end{gathered}
$$

The determinant of the Sylvester matrix does not depend on the parameter $t$.

Substituting $(32)$ in $(30)$ and further in 290 have

$$
F\left(t X_{0}, t X_{2}\right)=t^{n m} F\left(X_{0}, X_{2}\right) .
$$

Consequently, the resultant is a homogeneous polynomial of degree $n m$.
Theorem 1 admits a generalization. We have proved an analogous assertion for systems of equations of several variables, see [7, 16].

We transform system (3) and apply Bezout's theorem to its study.

In the equations of system (3) we proceed to homogeneous coordinates. Let

$$
\left\{\begin{array}{l}
x_{1}=X_{1} / X_{0} \\
x_{2}=X_{2} / X_{0}
\end{array}\right.
$$

After reducing the equations of the system to a polynomial form, we have

$$
\left\{\begin{array}{l}
X_{0}^{2 N+1} F_{1}\left(\frac{X_{1}}{X_{0}}, \frac{X_{2}}{X_{0}}, y_{1}\right)=\Phi_{1}\left(X_{0}: X_{1}: X_{2}\right)=0 \\
X_{0}^{2 N+1} F_{2}\left(\frac{X_{1}}{X_{0}}, \frac{X_{2}}{X_{0}}, y_{2}\right)=\Phi_{2}\left(X_{0}: X_{1}: X_{2}\right)=0 .
\end{array}\right.
$$

The coordinates $X_{0}, X_{1}, X_{2}$ are obviously projective coordinates.

The system (34) defines surjective mapping, $\Im: C^{2} \rightarrow C P^{2}$. The triple of complex numbers $\left(X_{0}: X_{1}: X_{2}\right)$ are the coordinates of the point and defines the point $p \in C P^{2}$ in the projective plane $C P^{2}$. The triple $\left(\lambda X_{0}: \lambda X_{1}: \lambda X_{2}\right)$ specifies the same point if $\lambda \neq 0$.

Therefore we have the following result.

Theorem 2. The system of polynomial equations

$$
\left\{\begin{array}{l}
\Phi_{1}\left(X_{0}: X_{1}: X_{2}\right)=0 \\
\Phi_{2}\left(X_{0}: X_{1}: X_{2}\right)=0
\end{array}\right.
$$

has in the projective plane $C P^{2}$, counting multiplicity, exactly $m \cdot n$ solutions, where, $m=\operatorname{deg} \Phi_{1}$, and $n=\operatorname{deg} \Phi_{2}$, if $\operatorname{gcd}\left(\Phi_{1}, \Phi_{2}\right)$ belongs to the coefficient field $C$.

Functions $\Phi_{1}=\Phi_{1}\left(X_{0}: X_{1}: X_{2}\right)$ and $\Phi_{2}=\Phi_{2}\left(X_{0}\right.$ : $\left.X_{1}: X_{2}\right)$ are homogeneous functions of degree $2 N+1$.

If, at least one of the coordinates of the point $p$ is equal to zero, say that this point is irregular. Otherwise, the point is called regular.

A straight line that consists of irregular points is called an irregular line.

The projective plane $C P^{2}$ has three irregular straight lines, which are given by the equations

$$
X_{0}=0, \quad X_{1}=0, \quad X_{2}=0 .
$$

The set of points $C P^{2}$ one of the coordinates, which is equal to the number $h \neq 0$, is called affine map on $C P^{2}$ and denoted by $A^{2}(h)$. The complement of a $C P^{2} \backslash A^{2}(h)$ consists of a one-dimensional complex projective subspace, which is called an infinitely distant line of the affine map, see for example [11, 15]. The infinitely distant line of any affine map $A^{2}(h)$ is evidently irregular.

In particular, if we put $X_{0}=1$, then the set of points $C P^{2}$ with coordinates $\left(1: X_{1}: X_{2}\right)$ will be 
affine map of $A^{2}(1)$, and the infinity of the straight line of this map will be given by equation $X_{0}=0$.

Consider the situation of general position, i.e. the source is not on the caustic. In this case, the Jacobian of the system of lens equations is not equal to zero.

Theorem 3. In a situation of general position (the Jacobian of the system of lens equations is not equal to zero), the number of point images in an $N$-point gravitational lens has parity opposite to the parity of the number $N$.

In the proof of Theorem 3 we use the following lemma.

Lemma 1. The number of irregular solutions of system (37), on line $X_{0}=0$, is $2 N$.

Proof. Using (37), we reduce the system to the form

$$
\left\{\begin{array}{l}
\left(X_{1}-X_{0} y_{1}\right) \prod_{i=1}^{N} H_{i} \\
\quad-X_{0}^{2} \sum_{j=1}^{N} m_{j}\left(X_{1}-X_{0} a_{j}\right) \prod_{i=1, i \neq j}^{N} H_{i}=0 \\
\left(X_{2}-X_{0} y_{2}\right) \prod_{i=1}^{N} H_{i} \\
\quad-X_{0}^{2} \sum_{j=1}^{N} m_{j}\left(X_{2}-X_{0} b_{j}\right) \prod_{i=1, i \neq j}^{N} H_{i}=0
\end{array}\right.
$$

where $H_{i}=\left(X_{1}-X_{0} a_{i}\right)^{2}+\left(X_{2}-X_{0} b_{i}\right)^{2}$.

Let $X_{0}=0$. We have

$$
\begin{aligned}
& \left\{\begin{array}{l}
X_{1} \prod_{i=1}^{N}\left(X_{1}^{2}+X_{2}^{2}\right)=0, \\
X_{2} \prod_{i=1}^{N}\left(X_{1}^{2}+X_{2}^{2}\right)=0
\end{array}\right. \\
& \Rightarrow\left\{\begin{array}{l}
X_{1}\left(X_{1}^{2}+X_{2}^{2}\right)^{N}=0, \\
X_{2}\left(X_{1}^{2}+X_{2}^{2}\right)^{N}=0
\end{array} \Rightarrow\left(X_{1}^{2}+X_{2}^{2}\right)^{N}=0\right. \\
& \Rightarrow X_{1}= \pm i X_{2} \Rightarrow\left\{\begin{array}{l}
X_{1}=c, \\
X_{2}= \pm i c .
\end{array}\right.
\end{aligned}
$$

Finally we have two $N$-fold solutions: $P_{1}=(0: a$ : $i c$ ) and $P_{2}=(0: a:-i c)$.

Proof of Theorem 2. For the degrees of the polynomials of systems (3) and (5) we have $\operatorname{deg} F_{1}=\operatorname{deg} F_{2}=$ $\operatorname{deg} \Phi_{1}=\operatorname{deg} \Phi_{2}=2 N+1$.

By Bezout's theorem, the system of equations 36 has $(2 N+1)^{2}$ solutions, which include an even number of $2 q$ complex conjugate solutions and $P=2 N$ irregular solutions. 36 ,

Therefore, the number of real solutions of system

$$
\begin{array}{r}
\operatorname{card}\left(\operatorname{real} V^{0}\left(F_{1}, F_{2}\right)\right)=(2 N+1)^{2}-2 q-P \\
=(2 N+1)^{2}-2 q-2 N \\
=4 N^{2}+2 N+1-2 q .
\end{array}
$$

From the fact that the restriction of the inverse mapping $\Im^{-1}: C P^{2} \rightarrow C^{2}$ to the affine map $A^{2}(1)$ is a bijection that is given by the equations $X_{0}=1$, $X_{1}=x_{1}, X_{2}=x_{2}$, we have

$$
\begin{array}{r}
\operatorname{card}\left(M^{0}\left(f_{1}, f_{2}\right)\right)=\operatorname{card}\left(\operatorname{real} V^{0}\left(F_{1}, F_{2}\right)\right)-N \\
=4 N^{2}+N+1-2 q .
\end{array}
$$

In a situation of general position, the point source is not on the caustic, therefore, all elements of the set real $V\left(f_{1}, f_{2}\right)$ are different.

In this case, each point of the set real $V\left(f_{1}, f_{2}\right)$ is, by definition, an image.

It follows from $(9)$ that the parity of the number of images is opposite to the parity of the number $N$.

Theorem 3 does not contradict the theorem on the oddness of the number of images in transparent lenses [9, 10].

Example 1. For a 1-point lens, the number of images is 2 , see $9,10,14$.

Example 2. For a 2-point lens, the number of images is 3 or 5 see [17.

\section{Conclusions}

Applying methods of algebraic geometry, we constructed an algorithm that separates images of dimensions 1 and 0 .

In the present paper, for an image of dimension 1 , it is proved that for single-point sources there exists a unique image of dimension 1-the Einstein ring; Einstein's ring is only in a single-point lens; the point source in other lenses does not have images of dimension 1 for $N>1$. For an image of dimension 0 , it is proved that in any $N$-point gravitational lens: there are a finite number of images; the parity of the number of images is always the opposite of the parity of the number $N$.

The assertion for the number of images of dimension 0 was proved by us earlier, see 18. In 18 , we used the geometric method of algebraic geometry-the Newton diagram. In the present paper all the assertions are proved algebraically. This opens the possibility, to use $N$-point gravitational lenses, not only approximate or numerical methods, but also computer algebra systems.

\section{A. Appendix}

Let $f(x, y)$ be a function of two variables, and $f(x, y)$, at the point $\left(x_{0}, y_{0}\right), n$-times continuous, differentiable function. Then Taylor's formula holds:

$$
f(x, y)=f\left(x_{0}, y_{0}\right)+\sum_{k=1}^{n} f^{(k)}\left(x-x_{0}, y-y_{0}\right)
$$


where

$$
\begin{aligned}
f^{(k)} & \left(x-x_{0}, y-y_{0}\right) \\
& =\sum_{k=1}^{n} C_{k}^{i} \frac{\partial^{k} f\left(x_{0}, y_{0}\right)}{\partial x^{k-i} \partial y^{i}}\left(x-x_{0}\right)^{k-i}\left(y-y_{0}\right)^{i},
\end{aligned}
$$

and $f(x, y)$ is the remainder term.

If $f(x, y)$ is a polynomial, and $m=\operatorname{deg} f(x, y)$, then $r_{n}(x, y)=0$ for all $n \geq m$.

Definition 1a. We say that a pair of numbers $x_{0}, y_{0}$ is a $s$-multiple solution of equation $f(x, y)=0$ if:

- $f\left(x_{0}, y_{0}\right)=0$;

- $f^{(i)}\left(x-x_{0}, y-y_{0}\right) \equiv 0, i=1,2, \ldots, s-1$;

- $f^{(s)}\left(x-x_{0}, y-y_{0}\right) \neq 0, s \leq n$ in some neighborhood of the point $\left(x_{0}, y_{0}\right)$; we will write this fact as $\operatorname{mult}\left(f\left(x_{0}, y_{0}\right)\right)=s$.

For example point $(0,0)$ is $s$-multiple solution of equation $f(x, y)=0$ if:

- $f(0,0)=0$;

- $f^{(i)}(x, y) \equiv 0, i=1,2, \ldots, s-1$;

- $f^{(s)}(x, y) \neq 0, s \leq n$ in some neighborhood of the point $(0,0)$.

Such a solution is called a $s$-multiple zero solution.

Definition 2a. Let the pair of numbers $x_{0}, y_{0}$ be a solution of the system of equations

$$
\left\{\begin{array}{l}
f(x, y)=0 \\
g(x, y)=0
\end{array}\right.
$$

and $q=\min \left(\operatorname{mult}\left(f\left(x_{0}, y_{0}\right)\right), \operatorname{mult}\left(g\left(x_{0}, y_{0}\right)\right)\right)$. The solution $x_{0}, y_{0}$ will be called $q$-multiple of the solution of the system of equations (44), while we will write $q=\min \left(\operatorname{mult}(f, g)\left(x_{0}, y_{0}\right)\right)$.

The concept of a multiple solution of a system of equations can obviously be extended to systems with an arbitrary number of equations from several variables.

The resultant of polynomials is one of the basic concepts of classical algebraic geometry.

In the modern literature [12, 19, 20, the resultant of polynomials is usually defined as follows.

Definition 3a. Let $K$-arbitrary field, $f(x)$ and $g(x)$ polynomials in $K[x]$. The resultant $R(f, g)$ of polynomials $f(x)$ and $g(x)$ is called an element in the field $K$, defined by the formula

$$
R(f, g)=a_{0}^{n} b_{0}^{m} \prod_{i=0}^{n} \prod_{j=0}^{m}\left(\alpha_{i}-\beta_{j}\right),
$$

where $\alpha_{i}, \beta_{i}$ are roots of polynomials $f(x)=$ $\sum_{i=0}^{n} a_{i} x^{n-i}$ and $g(x)=\sum_{j=0}^{m} b_{j} x^{m-j}$, correspondingly, with the highest coefficients, $a_{0}, b_{0}$ such that $a_{0} \neq 0, b_{0} \neq 0$.
Let the roots of the polynomials $f(x)$ and $g(x)$ be known. To calculate their resultant, one can use formula (45).

If we know only the coefficients of these polynomials, then we can use the Sylvester matrix to calculate their resultant. The Sylvester matrix is a block matrix of two blocks. Each block has one ribbon matrix. We have a definition of the Sylvester matrix.

Definition 4a. Matrix Sylvester for polynomials $f(x)=\sum_{i=0}^{n} a_{i} x^{n-i}$ and $g(x)=\sum_{j=0}^{m} b_{j} x^{m-j}$, we call a square matrix $S=S(f, g)$ of order $n+m$ with elements $s_{i j}$ defined by the formula

$$
s_{i j}=\left\{\begin{aligned}
a_{j-i}, & \text { if } 0 \leq j-i \leq n, \\
& i=1, \ldots, m, j=1, \ldots, n+m, \\
b_{j-i+m}, & \text { if } 0 \leq j-i+m \leq n, \\
& i=m+1, \ldots, m+n, \\
& j=1, \ldots, n+m, \\
0, & \text { for other } i, j,
\end{aligned}\right.
$$

i.e.,

$$
S(f, g)=\left[s_{i j}\right]=\left\{\begin{array}{ccccccc}
a_{0} & a_{1} & \cdots & a_{n} & 0 & \cdots & 0 \\
0 & a_{0} & a_{1} & \cdots & a_{n} & 0 & 0 \\
& & \ddots & \ddots & \ddots & \\
0 & \cdots & 0 & a_{0} & a_{1} & \cdots & a_{n} \\
b_{0} & b_{1} & \cdots & b_{m} & 0 & \cdots & 0 \\
0 & b_{0} & b_{1} & \cdots & b_{m} & 0 & 0 \\
& & \ddots & \ddots & & \ddots & \\
0 & \cdots & 0 & b_{0} & b_{1} & \cdots & b_{m}
\end{array}\right] .
$$

The resultant polynomials $R(f, g)$ and the Sylvester matrix $\operatorname{Sul}(f, g)$ are connected by the following theorem.

Theorem 1a. The resultant $R(f, g)$ of the polynomials $f$ and $g$ is equal to the determinant of Sylvester matrix these polynomials, i.e., $R(f, g)=S(f, g)$.

For the proof see, e.g., [19, 20].

Theorem 2a. Polynomials $f$ and $g$ have a common root if and only if

$$
R(f, g)=0
$$

For the proof see, e.g., [13].

Theorem 3a (Bezout). The number of intersection points of plane curves $\Phi_{1}$ and $\Phi_{2}$ (counted taking into account the multiplicity) is equal to $\mathrm{nm}$, where $m=\operatorname{deg} \Phi_{1}$, and $n=\operatorname{deg} \Phi_{2}$, if the curves:

- do not have common components;

- are defined over an algebraically closed field;

- are considered on the projective plane.

For the proof see, e.g., [12]. 
Theorem 4a. Let $f\left(x_{1}, x_{2}\right)=\sum_{i, j=0}^{i+j \leq n} f_{i j} x_{1}^{i} x_{2}^{j}$ and $g\left(x_{1}, x_{2}\right)=\sum_{i, j=0}^{i+j \leq m} g_{i j} x_{1}^{i} x_{2}^{j}$ be polynomials. Let their coefficients be such that $f_{n 0} \neq 0, f_{0 n} \neq 0$, $g_{m 0} \neq 0, g_{0 m} \neq 0$. Then

$$
\begin{aligned}
\operatorname{deg} R_{1}( & \left.f\left(x_{1}, x_{2}\right), g\left(x_{1}, x_{2}\right)\right) \\
& =\operatorname{deg} f\left(x_{1}, x_{2}\right) \operatorname{deg} g\left(x_{1}, x_{2}\right)=n m .
\end{aligned}
$$

For the proof see, e.g., [12].

Theorem 5a. The polynoms $F_{1}\left(x_{1}, x_{2}\right), F_{2}\left(x_{1}, x_{2}\right)$ have a non-trivial common component if and only if $R_{1}\left(F_{1}\left(x_{1}, x_{2}\right), F_{1}\left(x_{1}, x_{2}\right)\right)$ or $R_{2}\left(F_{1}\left(x_{1}, x_{2}\right)\right.$, $\left.F_{1}\left(x_{1}, x_{2}\right)\right)$.

For the proof see, e.g., [12].

Definition 5a. A formal sum $G=G\left(x_{1}, x_{2}\right)$ of the form $G=\sum_{i, j=0}^{i+j \leq n} g_{i j} x_{1}^{i} x_{2}^{j}$ is called a polynomial $n$ form of variables $x_{1}, x_{2}$ over the field $K$. that is, $G$ is a polynomial of degree $n$ in variables $x_{1}, x_{2}$ with indefinite coefficients $g_{i j}$ in the field $K$.

The expression "the function will be sought in the form of a $n$-form" is usually understood as a procedure for determining the undetermined coefficients of a given $n$-form.

Theorem 6a (Criterion of non-decomposability). Let $F$ be a polynomial in the variables over the field of complex numbers and. Let, and let be the -form of the variables $x_{1}$ and $x_{2}$ over the field of complex numbers. Let and be the resultants with respect to the variables and respectively.

The polynomial $F$ is not decomposable if and only if the systems of equations

$$
\begin{aligned}
& \frac{\partial^{i}}{\partial x_{1}^{i}} R_{1}\left(F\left(x_{1}, x_{2}\right), G\left(x_{1}, x_{2}\right)\right)=0, \\
& \frac{\partial^{i}}{\partial x_{2}^{i}} R_{2}\left(F\left(x_{1}, x_{2}\right), G\left(x_{1}, x_{2}\right)\right)=0,
\end{aligned}
$$

where $i=1, \ldots, m, m=\operatorname{deg} R_{1}(F, G)$, have only zero solutions.

The system (50) is considered with respect to the undetermined coefficients $g_{i j} n$-form $G$, as with respect to unknown variables.

For the proof see, e.g., [7].

The theorem admits a generalization to the case of systems of equations of several variables see [7, 16].

The criterion was formulated and proved by the authors earlier [7].

\section{REFERENCES}

[1] E. Y. Bannikova, A. T. Kotvytskiy. Three Einstein rings: explicit solution and numerical simulation. MNRAS 445(4):4435-4442, 2014. DOI:10.1093/mnras/stu2068

[2] A. T. Kotvytskiy. Gravitational lensing by straight cosmic strings. TMP 184(1):160-174, 2015. DOI:10.1007/s11232-015-0315-x
[3] A. Cassan. An alternative parameterisation for binary-lens caustic-crossing events. Astronomy and Astrophysics 491(2):587-595, 2008. DOI:10.1051/0004-6361:200809795

[4] P. Schneider, A. Weiss. The two-point-mass lens: detailed investigation of a special asymmetric gravitational lens. Astronomy and Astrophysics 164(2):237-259, 1986.

[5] A. T. Kotvytskiy, S. D. Bronza, K. D. Nerushenko, V. Y. Shablenko. Matematychnii zmist kiltsia Einshteina ta umovy yogo vynyknennja. doslidzhennya uzahal'nenyh umov[in ukrainian]. In Zbirnyk naukovyx prac' VI-i Mizhrehional'noi naukovo-praktychnoi konferencii "Astronomiya $i$ s'ogodennya", pp. 198-213. Vinnytsia, 2017.

[6] A. T. Kotvytskiy, S. D. Bronza, S. R. Vovk. Estimating the number of images N-point gravitational lenses algebraic geometry methods. Visnyk KhNU, serija "Fizyka" 24:55-59, 2016.

[7] S. D. Bronza, A. T. Kotvytskiy. Mathematical bases of the theory of N-point gravitational lenses. Part 1. Elements of algebraic geometry. Visnyk $K h N U$, seriya "Fizyka" 26:6-32, 2017.

[8] A. T. Kotvytskiy, S. D. Bronza. Mathematical bases of the theory of N-point lenses. Odessa Astronomical Publications 29:31-33, 2016. DOI:0.18524/1810-4215.2016.29.84958

[9] A. F. Zakharov. Gravitacionnye linzy i mikrolinzy [in Russian]. Janus-K, Moscow, 1997.

[10] P. Schneider, J. Ehlers, E. Falco. Gravitational lenses. Second printing. Springer-Verlag Berlin Heidelberg, 1999.

[11] I. V. Arzhantcev. Bazisy Grebnera i sistemy algebraicheskih uravnenij [in Russian]. MCNMO, Moscow, 2003.

[12] R. J. Walker. Algebraic curves. Springer-Verlag New York, 1978.

[13] E. Kalinina, A. J. Uteshev. Teoria iskluchenij [in Russian]. NII chimii SPbGU Saint Petersburg, 2002.

[14] P. V. Bliokh, A. A. Minakov. Gravitational Lenses [in Russian]. Naukova Dumka, Kiev, 1989.

[15] M. Reid. Undergraduate Algebraic Geometry. Math Inst., University of Warwick, 2013.

[16] S. D. Bronza. Kriteriy neprivodimosti mnogochlenov ot dvuh peremennyih nad polem kompleksnih chisel[in russian]. In Zbirnyk naukovyx prac', pp. 114-115. UkrDUZT, 2016.

[17] S. H. Phie. Infimum microlensing amplification of the maximum number of images of N-point lens systems. The astrophysical journal 484(1):63-69, 1997. DOI:10.1086/304336

[18] A. T. Kotvytskiy, S. D. Bronza, V. Y. Shablenko. Correlation of the number of images of an N-point gravitational lens and the number of solutions of its system. In Astronomy and beyond: astrophysics, cosmology, cosmomicrophysics, astroparticle physics, radioastronomy and astrobiology, p. 12. Odessa International Astronomical Gamow Conference-School, 2017.

[19] B. L. van der Waerden. Modern Algebra I, II. New York:Frederic Ungar Publishing Co., 1950.

[20] S. Lang. Algebra. Revised Third Edition. Columbia University. New York, 1965. 